\title{
Asymptotic Scaling, Casimir Scaling, and Center Vortices
}

\author{
M. Faber ${ }^{\mathrm{a}}$, J. Greensite ${ }^{\mathrm{b}}$, Šs. Olejník ${ }^{\mathrm{c}}$

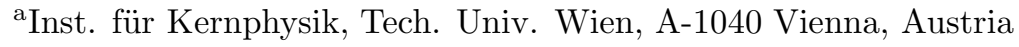 \\ bThe Niels Bohr Institute, DK-2100 Copenhagen Ø, Denmark \\ ${ }^{\mathrm{c}}$ Inst. of Phys., Slovak Acad. of Sci., SK-842 28 Bratislava, Slovakia
}

We report on two recent developments in the center vortex theory of confinement: (i) the asymptotic scaling of the vortex density, as measured in Monte Carlo simulations; and (ii) an explanation of Casimir scaling and the adjoint string tension, in terms of the center vortex mechanism.

\section{Introduction}

Center vortices in $\mathrm{SU}(2)$ lattice gauge theory are detected on thermalized lattices by fixing to the maximal center gauge, in which the quantity

$R=\sum_{x} \sum_{\mu}\left(\operatorname{Tr}\left[U_{\mu}(x)\right]\right)^{2}$

is maximized, and then mapping link variables onto a $Z_{2}$ configuration via center projection

$U_{\mu}(x) \rightarrow Z_{\mu}(x)=\operatorname{sign} \operatorname{Tr}\left[U_{\mu}(x)\right]$

The excitations of a $Z_{2}$ lattice gauge field are $Z_{2}$ vortices, which we term "projection-vortices" or "P-vortices." A plaquette is pierced by a $\mathrm{P}$-vortex if, in the center-projected lattice, the plaquette has the value -1 . We then define the "vortexlimited" Wilson loops $W_{n}(x)$ to be loops on the unprojected lattice evaluated when exactly $n$ Pvortices, in the center-projected lattice, pierce the minimal area of the loop. It is then found that: (i) $\mathrm{P}$-vortices on the projected lattice locate (thick) center vortices on the unprojected lattice, as deduced from the asymptotic behavior of the ratios $W_{n}(C) / W_{0}(C) \rightarrow(-1)^{n}$; (ii) no vortices correlates with no confinement, i.e. $\chi_{0}(I, J) \rightarrow 0$; (iii) vortices, by themselves, account for the full asymptotic string tension obtained by standard methods, with the potential linear beginning at

\footnotetext{
*Talk presented by J. Greensite. Work supported by Carlsbergfondet, and by the U.S. Department of Energy under Grant No. DE-FG03-92ER40711.
}

one lattice spacing. These results, together with further references, are collected in ref. [1]. It was also found that, upon abelian projection, center vortices appear as monopole-antimonopole chains. The action of monopole cubes is almost entirely concentrated in plaquettes pierced by $\mathrm{P}$ vortices, and the (unprojected) action distribution of monopole cubes is not much different from any other cube pierced by a P-vortex, c.f. [2]. For another approach to vortices, cf. [3].

In this talk I would like to report on two further aspects of center vortices: asymptotic scaling and Casimir scaling.

\section{Vortex Density: Asymptotic Scaling}

The result that the density of center vortices scales according to the asymptotic freedom prediction was first obtained by Langfeld et al. [4], using a slightly different version of maximal center gauge. Here I will only display the results of our group, using the gauge obtained by maximizing (这). Define $N_{\text {vor }}$ to be the total number of plaquettes pierced by P-vortices, and $N_{T}$ the total number of plaquettes on the lattice. Then

$p=\frac{N_{\text {vor }}}{N_{T}}=\frac{\text { Total Vortex Area }}{6 \times \text { Total Volume }} a^{2}=\frac{1}{6} \rho a^{2}$

where $\rho$ is the center vortex density (vortex area/unit volume) in physical units. Then, according to asymptotic freedom

$p=\frac{\rho}{6 \Lambda^{2}}\left(\frac{6 \pi^{2}}{11} \beta\right)^{102 / 121} \exp \left[-\frac{6 \pi^{2}}{11} \beta\right]$ 


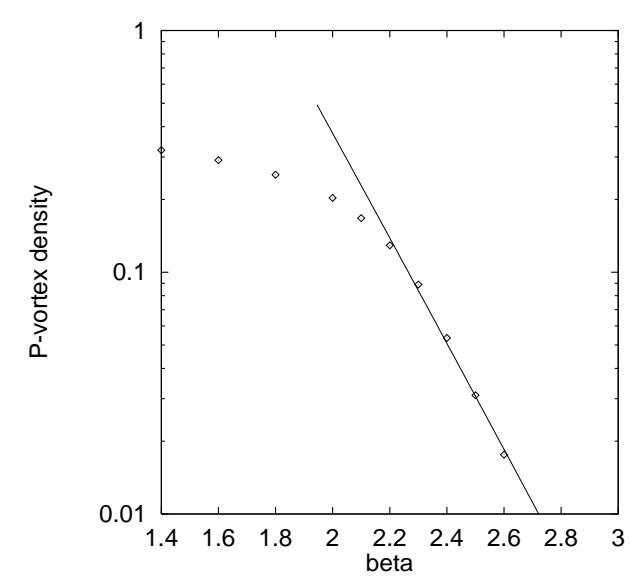

Figure 1. Vortex density vs. coupling.

Figure 1 is a plot of $p$ vs. $\beta$. The straight line is the asymptotic freedom result, with $\sqrt{\rho / 6 \Lambda^{2}}=$ 50. Note that the scaling line has the slope appropriate to a density of surfaces. The scaling lines for densities of pointlike objects (instantons) or linelike objects (monopole loops) would have very different slopes.

\section{Casimir Scaling}

Our proposal is that the Casimir scaling of string tensions in the intermediate distance regime is an effect due to the finite thickness of center vortices [5] (see also [6]). A model of those effects for SU(2) gauge theory, presented in more detail in ref. [5], is the following: If a vortex pierces the minimal area of a planar Wilson loop, its effect is represented by the insertion of a group element $G$

$G=S \exp \left[i \alpha_{C}(x) \sigma_{3} / 2\right] S^{\dagger}$

into the product of (zero-vortex) link variables around loop C. It is assumed that $S$ is a randomly distributed group element, and $\alpha_{C}(x) \in[0,2 \pi]$ depends on the location at which the vortex pierces the minimal area of the loop. If the crosssection of the vortex "core", where the vortex crosses the plane of the loop, is entirely contained in the minimal area, then $G=-I(\alpha=2 \pi)$, while if the vortex cross section is entirely exterior to the minimal area, then $G=+I(\alpha=0)$. We have $G \neq \pm I$ if the vortex somewhere overlaps the loop perimeter. If we let $f$ denote the probability that the middle of a vortex pierces any given plaquette, and further assume that the positions of vortices piercing plaquettes in a plane are uncorrelated, then the vortex contribution to the heavy-quark potential is found to be

$$
\begin{aligned}
V_{j}(R) & =-\sum_{n=-\infty}^{\infty} \ln \left\{(1-f)+f \mathcal{G}_{j}\left[\alpha_{R}\left(\mathrm{x}_{n}\right)\right]\right\} \\
\mathcal{G}_{j}[\alpha] & =\frac{1}{2 j+1} \sum_{m=-j}^{j} \cos (\alpha m)
\end{aligned}
$$

where $j$ denotes the $\mathrm{SU}(2)$ group representation of the heavy quark charges. It can then be shown that the asymptotic string tension is $\sigma_{j}=-\ln (1-2 f)$ for $j$ =half-integer, and $\sigma=0$ for $j=$ integer. For small $R$, on the other hand, where $\alpha_{R}(x) \ll 2 \pi$, we find

$V_{j}(R)=\left\{\frac{f}{6} \sum_{n=-\infty}^{\infty} \alpha_{R}^{2}\left(\mathrm{x}_{n}\right)\right\} j(j+1)$

which is proportional to the $\mathrm{SU}(2)$ Casimir (the result readily generalizes to $\mathrm{SU}(\mathrm{N})$ ). To go further, one needs some knowledge of $\alpha_{R}(x)$. The behavior of this function in the $R \rightarrow 0, R \rightarrow \infty$, and $x \rightarrow \pm \infty$ limits is known, and most reasonable ansätze for $\alpha_{R}(x)$ do indeed display a Casimir-scaling region at intermediate distances, where the potential is roughly linear, and also roughly proportional to $j(j+1)$ [5].

If this explanation of Casimir-scaling is correct, then the string tension of zero-vortex Wilson loops $W_{0}^{a d j}(C)$ in the adjoint representation should disappear in the Casimir-scaling regime. In principle this is easy to test: we just compare Creutz ratios $\chi_{0}^{a d j}(I, J)$ with $\chi^{a d j}(I, J)$. In practice, however, the VEV of adjoint loops is very small, and some reduction in noise due to highfrequency fluctuations is essential. This we have done by applying the constrained cooling procedure of ref. [7]. Figure 目 is an illustration of Casimir scaling on the cooled lattice $(\beta=2.3,10$ cooling steps). The solid line shows Creutz ratios 


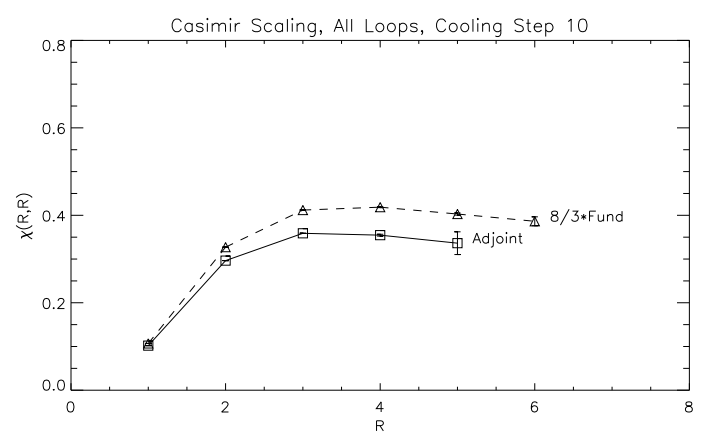

Figure 2. Adjoint and fundamental $(\times 8 / 3)$ Creutz ratios, $\beta=2.3$.

$\chi(R, R)$ in the adjoint representation (solid line), and in the fundamental representation rescaled by $8 / 3$ (dashed line). If Casimir scaling were exact, the two lines would coincide. In both adjoint and fundamental, the string tension seems to stabilize around $R=3$. Figure 3 shows the corresponding quantities extracted from zero-vortex Wilson loops. In this case we notice that Casimir-scaling is nearly exact, and there is evidently no string tension; the Creutz ratios are clearly tending towards zero in both the adjoint and fundamental representations. It appears that when center vortices are excluded from the interior of Wilson loops, both the fundamental and adjoint string tensions disappear. We view this as evidence in favor of a vortex origin of the adjoint string tension, in the Casimir-scaling regime. Further details may be found in ref. [8].

\section{Conclusions}

The asymptotic scaling of the center vortex density strengthens our conviction that these surface-like objects identified by center projection in maximal center gauge are physical objects. Asymptotic scaling is not the only evidence of physicality; we have previously seen that large Wilson loops linked to these objects pick up an



Figure 3. Zero-vortex adjoint Creutz ratios, $\beta=$ 2.3 .

extra factor of -1 迎 (which allows us to identify them as center vortices), and that the vortices themselves are associated with surfaces of anomalously high action-density [2]. But certainly the scaling of the vortex density is an important part of the picture.

We have also presented an explanation of the adjoint string tension, in the context of the center vortex mechanism, and displayed some numerical evidence that the existence of an adjoint tension correlates with the presence of vortices linking the loop. This provides, we believe, a plausible answer to the question "What about Casimir Scaling?" in connection with center vortices.

\section{REFERENCES}

1. L. Del Debbio et al., hep-lat/9801027.

2. L. Del Debbio et al., hep-lat/9708023.

3. T. Kovács and E. Tomboulis, hep-lat/9711009.

4. K. Langfeld et al., Phys. Lett. B419 (1998) 317 ; hep-lat/9710068.

5. M. Faber et al., Phys. Rev. D57 (1998) 2603; hep-lat/9710039.

6. J. Cornwall, hep-th/9712248.

7. M. Campostrini et al., Phys. Lett. B225 (1989) 403.

8. M. Faber et al., hep-lat/9807008. 